Voix et Images

voixetimages

\title{
Perspectives contemporaines sur la chanson francophone
}

\section{Danielle Tremblay}

Volume 14, numéro 2 (41), hiver 1989

L’édition littéraire au Québec

URI : https://id.erudit.org/iderudit/200781ar

DOI : https://doi.org/10.7202/200781ar

Aller au sommaire du numéro

\section{Éditeur(s)}

Université du Québec à Montréal

\section{ISSN}

0318-9201 (imprimé)

1705-933X (numérique)

Découvrir la revue

\section{Citer cet article}

Tremblay, D. (1989). Perspectives contemporaines sur la chanson francophone. Voix et Images, 14(2), 333-336. https://doi.org/10.7202/200781ar d'utilisation que vous pouvez consulter en ligne.

https://apropos.erudit.org/fr/usagers/politique-dutilisation/ 


\section{Perspectives contemporaines sur la chanson francophone}

\section{par Danielle Tremblay, Université de Sherbrooke}

La chanson populaire attire de plus en plus l'attention des universitaires. Elle n'est plus tout à fait considérée comme un art «bâtard» et l'on découvre sa valcur profonde dans la communication culturelle. Deux ouvrages récents explorent plusieurs dimensions de cet art populaire dans le contexte des socićtés occidentales contemporaines, avec une insistance particulière sur le Québec. Des approches thćoriques et méthodologiques variées, mais complémentaires, élargissent le débat pour tout lectcur intrigué par les résonances sociales, esthétiques et ćconomiques du phénomène de la chanson.

La Chanson dans tous ses états ${ }^{1}$ cst la troisième publication d'un collectif de chercheurs ratlaché à l'Université de Sherbrooke, sous la direction de Robert Giroux. Dans la lignée des Aires de la chanson québécoise paru en 1984 et de la 
Chanson en question(s) paru en 1985, ce recueil d'articles touche plusieurs aspects du phénomène: l'histoire sociale, l'histoire économique, les représentations symboliques et culturelles. Le nombre et la qualité des travaux démontrent une très nette progression, d'un volet à l'autre, chez les collaborateurs. Dans ce recueil, Renée-Berthe Drapeau, d'une plume alerte mais rigoureuse, couvre l'évolution de trois genres différents en musique et chanson populaires dans le Québec de l'entre-deux-guerres: «Chant, chanson et chansonnette au Québec: 1920-1950». Sylvie Faure poursuit une analyse sociologique et esthétique à propos d'un mouvement très circonscrit dans la musique populaire: «Le mouvement punk, un processus de séduction». Elle explique comment ce phénomène musical et culturel est passé d'une phase de négation sociale à une récupération par la mode et la consommation de masse. René Charest et Robert Giroux, dans «La chanson populaire en Estrie», montrent le dynamisme et la variété de la production de la chanson estrienne à travers l'histoire de la culture régionale. Certaines analyses sont plus théoriques. Celle de Jacques Julien, «La fonction conative dans la chanson populaire», s'appuie sur les sciences du langage pour étudier le pouvoir de persuasion du verbe, de la gestuelle et du son dans la pratique culturelle de la chanson.

Les instruments méthodologiques importants pour l'histoire de la chanson ne sont pas négligés. La recherche à partir des «sources techniques» de la production fait l'objet d'un intéressant article par Jean-Jacques Schira et Robert Giroux: «La discographie: un instrument de mesure de la popularité au Québec 1898-1959». Enfin, certains textes nous tiennent en alerte en passant au crible de la critique plusieurs phénomènes rapportés à l'industrie culturelle. Bruno Roy signe un texte sur la censure socio-politique: «Ils chantent, qu'ils paient! ou De la censure». Yves Laberge et Réal LaRochelle proposent des textes sur l'essor du vidéo-clip, ses conditions socio-économiques et ses répercussions esthétiques sur la pratique de la chanson. Le discours actuel des intervenants culturels, critiques, chercheurs et professeurs, fait lui-même l'objet d'une synthèse et d'une réflexion par Robert Giroux.

Ce programme comporte autant d'éléments fascinants que frustrants sur le plan de la recherche. La générosité du propos, la pertinence générale des points de vue peuvent alimenter la curiosité et provoquer de plus amples recherches. On sent cependant une certaine fragilité dans la synthèse de toutes ces recherches et de tous ces commentaires. J'accepte très bien que le débat soit ouvert à plusieurs thèmes et à plusieurs discours, avec toutes les possibilités de la chanson-performance comme fil conducteur. Ce qui déçoit un peu tient à l'inégalité des expériences et donc des performances de recherche. C'est sans doute le lot de ce type d'ouvrage collectif.

Or, trois conditions sont essentielles pour mener à bien une recherche et la présenter de façon révélatrice. Il faut poser clairement le problème, justifier les méthodes d'analyse et explorer les résultats pour en faire jaillir des réflexions éloquentes. Ainsi, certains articles apparaissent d'emblée comme des condensés de recherches élaborées: citons comme exemple «La POPularisation de l'opéra par les industries culturelles» où Réal LaRochelle analyse le cas de Maria Callas. En revanche, certains textes donnent l'impression de plans de recherche, 
intéressants mais incomplets à un ou plusieurs niveaux. Par exemple, l'article qui traite des productions pour enfants de Nathalie Simard et de Passe-partout souffre d'une interprétation superficielle, malgré des commentaires intéressants de Manon Poulin sur les perceptions artistiques propres à ce groupe d'âge. De plus, quelques articles sacrifient parfois la cohérence historique à la polémique: en lisant celui de Bruno Roy sur la censure, par exemple, on se retrouve devant une série de «flashes» qui rappellent des moments forts plus qu'ils n'en apprennent au lecteur sur les mécanismes de la censure elle-même.

Évidemment, il est difficile de rassembler des volontés de recherche dans un domaine encore si éclaté. Il s'agit ici d'une tentative de coordonner des travaux aux cheminements très divers, mais on se prend à espérer une plus grande concertation entre les personnes intéressées. En outre, on souhaiterait que davantage de temps et de ressources soient consacrés à la réalité culturelle québécoise, si riche sur le plan des arts populaires et de la chanson en particulicr. Plusieurs des co-auteurs abonderaient dans le même sens

Le livre de Denis Bégin, la Chanson québécoise ${ }^{2}$, répond essentiellement à des objectifs pédagogiques: il s'adresse donc à un public plus large que la communauté des cherchcurs et des mélomanes. Il se compose de trois parties distinctes: une description du contexte historique (très brève), une présentation analytique des paroles et musiques de vingt-quatre chansons et une abondante nomenclature de sources de renseignements sur la chanson québécoise.

La partie historique, intitulée «La chanson et son milieu», couvre la période 1920-1986. Elle est divisée en deux chapitres: «La société québécoise» (le social, le politique, l'économique, le religieux) et «La chanson québécoise» (le terroir, la chanson populaire, l'art lyrique). S'ajoute un appendice, «La chanson-produit». On peut discuter ces divisions assez arbitraires et artificielles du champ historique. Malgré une quantité appréciable d'informations, il est regrettable de constater la maladresse de la synthèse en plusieurs endroits. Cela se traduit surtout par la simplification abusive de grands pans de l'histoire sociale et culturelle. Par exemple, l'argumentation rapide et hachée du chapitre intitulé «La chanson qućbćcoise» élimine toute continuité entre l'avènement des médias modernes, l'évolution des différents styles de chanson populaire et leurs échanges nécessaires au développement d'unc chanson québćcoise autonome. La chanson se retrouve «plaquée» sur une réalité sociale, politique et économique faite trop souvent de lieux communs. Les chansons symboliques qui accompagnent le texte sont souvent isolées du contexte de leur création. Elles semblent là davantage pour appuyer une interprétation de l'histoire que pour favoriser la compréhension d'une époque.

La partie la plus importante (209 pages) analyse vingt-quatre chansons célèbres. L'auteur, dans ses notes complémentaires, dit s'être servi des modèles d'analyse sémiotique de A.J. Greimas, du groupe $\mu$ («Mu») et de Gérard Genctte. Ces illustrations ont sans aucun doute été rédigées avec conscience et assiduité, mais les structures thématiques et rhétoriques de chansons ne s'analysent pas comme des poèmes hermétiques. Des textes composés avant tout pour ĉtre mis en musique, inséparables de ce contexte, appellent une autre perception. L'analyste insiste plutôt lourdement sur les références directes du texte, quand il 
ne s'acharne pas sur certains poncifs "poétiques». Les explications réduisent souvent la richesse sémantique que pourraient percevoir les auditeurs et les auditrices; elles répètent des affirmations facilement comprises à la première écoute de la chanson. Le plus grave est que, dans toute cette partie du livre, on constate un ravalement systématique de la compréhension des Québécois, jeunes ou moins jeunes. Peut-être est-ce moins le fait d'une mauvaise volonté que d'une vision myope de l'enseignement des lettres en général et en particulier de l'art populaire qu'est la chanson.

Le choix des chansons elles-mêmes apparait approprié, mais un peu court. Une production importante d'auteurs-compositeurs-interprètes parmi les plus marquants des années 70 et 80 a été négligée: citons Claude Dubois, Diane Dufresne, Plume Latraverse. D'ailleurs, quelques lignes seulement leur sont consacrées dans l'historique. Les explications des notes sont révélatrices d'un certain point de vue critique qui recherche le meilleur «goût littéraire». Se pourrait-il que les classiques de la chanson soient toujours définis au nom des mêmes critères respectables?

La partie la plus intéressante du livre concerne la documentation de cinquante-cinq pages assemblée par Richard Perreault. Elle comprend une discographie qui a le double mérite de l'équilibre et de l'accessibilité, une bibliographie des recueils de paroles et des cahiers de paroles/musiques disponibles, et une liste des principaux distributeurs de disques québécois.

En résumé, la Chanson québécoise possède des faiblesses inhérentes à son genre, en s'appropriant une démarche pédagogique trop restreinte et, disons-le, proche de la prétention. Le livre contient cependant des renseignements précieux pour les non-initiés qui auront tout le loisir de pousser plus à fond leurs investigations, à l'aide de la partie documentaire qui referme en beauté l'ouvrage.

Je conclus ce compte rendu en me réjouissant d'abord de l'amélioration du statut de la chanson, à la fois comme témoignage culturel et comme pratique artistique. J'estime cependant que la diversité des perspectives ne doit pas sacrifier à certaines exigences. Il faut rejeter les attitudes désuètes envers la chanson populaire et ses publics et viser la meilleure compréhension possible des différents liens entre la chanson et le monde où elle s'inscrit.

1 Robert Giroux, la Chanson dans tous ses états, Montréal, Tryptique, 1987, $238 \mathrm{p}$.

2 Denis Bégin, la Chanson québécoise, Cap-de-la-Madeleine, Réseau U, 1987, 354 p. 